\title{
Spontaneous magnetostriction in the Gd-Y system: analysis of phase transformations
}

\author{
A. B. Beznosov, E. L. Fertman, V. V. Eremenko, and P. P. Pal-Val \\ B.Verkin Institute for Low Temperature Physics and Engineering of the National Academy of Sciences of Ukraine \\ 47 Lenin Ave., Kharkov 61164, Ukraine \\ E-mail: beznosov@ilt.kharkov.ua \\ V. P. Popov and N. N. Chebotayev \\ V. N. Karazin Kharkov National University, 4 Svoboda Sq., Kharkov 61077, Ukraine
}

Received December 11, 2000

\begin{abstract}
The transformations of magnetic and lattice subsystems states of $\mathrm{Gd}_{100-x} \mathrm{Y}_{x}(x=0,5.5,7.5,10.2)$ alloys have been studied at temperatures $5-370 \mathrm{~K}$ in magnetic fields up to $4 \mathrm{kOe}$. The temperature dependences of elastic modules, thermal expansion, low field magnetization and magnetic anisotropy parameters were obtained. The linear spontaneous striction $\Delta L(T) / L=\xi \eta^{2}$ was analized, and the effective order parameters $\eta$ and magnetostrictive parameters $\xi$ were evaluated for the four magnetic phases (canted ferromagnetic, deforming ferromagnetic helix, ferromagnetic helix, and the ferromagnetic cone, respectively): $\xi_{c f}=2 \cdot 2 \cdot 10^{-5}, \xi_{d h}=-5.3 \cdot 10^{-5}, \xi_{f h}=7 \cdot 1 \cdot 10^{-5}, \xi_{f c}=1.4 \cdot 10^{-3}$.

PACS: $75.30 . \mathrm{Kz}, \mathbf{7 5 . 5 0 . - y}$
\end{abstract}

\section{Introduction}

Rare-earth metals and their alloys are of permanent great interest both in fundamental and applied science. Due to their unusual electronic structure rare earth metals and alloys demonstrate unique magnetic properties and a number of magnetic phase transitions. The Gd-Y alloys attract attention in view of the competing ferromagnetic order favored by Gd and antiferromagnetic order found in the Y-rich alloys.

Between 226 and $292.5 \mathrm{~K}$ gadolinium is a collinear ferromagnet with the easy magnetization axis directed along the $\mathbf{c}$ axis of hexagonal crystal structure. At $T_{s}=226 \mathrm{~K}$ there is the second-order spinreorientational phase transition. In the alloy $\mathrm{Gd}_{80} \mathrm{Y}_{20}$ only the ferromagnetic structure is found with the magnetic moments tilted by the angle $70^{\circ}$ from hexagonal $\mathbf{c}$ axis at all the temperatures lower than the Curie temperature $T_{C}$. The antiferromagnetic helical phase appears in alloys containing about 30 at.\% Y. The alloys with $30-40$ at.\% Y exhibit the antiferromagmetic structure as well as the ferromagnetic one depending on the temperature region. In the alloys with higher $\mathrm{Y}$ content only the antiferromagnetic structures are observed [1-3].
Recent studies of $\mathrm{Gd}-\mathrm{Y}$ alloys [4,5] have revealed their complex magnetic behavior, which differs substantially from that of pure Gd [6] as well as of $\mathrm{Gd}-\mathrm{Y}$ alloys with 20 at.\% and more $\mathrm{Y}$ content, and a new magnetic phases (probably, sorts of the ferromagnetic helix) in weakly diluted $\mathrm{Gd}-\mathrm{Y}$ alloys were assumed.

The goal of the present paper was to study the temperature behavior both of the magnetic system and the crystal lattice of polycrystalline $\mathrm{Gd}-\mathrm{Y}$ alloys with yttrium content $0-12$ at.\% over a wide temperature range $(5-300 \mathrm{~K})$ and to evaluate qualitatively the effective magnetic order parameters and the spontaneous magnetostriction coefficients.

\section{Research technique}

The polycrystalline substitution alloys $\mathrm{Gd}_{100-x} \mathrm{Y}_{x}$ $(x=0,5.5,7.5,10.2,12.0)$ were prepared from the raw materials of $99.88 \%(\mathrm{Gd})$ and $99.96(\mathrm{Y})$ purity at the OKMZ GIREDMET (Podolsk, Russia). The chemical analysis has shown following amount of impurities (in \%): $\mathrm{Tb}<0.05, \mathrm{Cu}<0.01, \mathrm{Fe} \approx 0.01$, $\mathrm{Si}<0.01, \mathrm{H}<0.01, \mathrm{~N}<0.05, \mathrm{~F} \approx 0.01, \mathrm{C}<0.01$. The specimens were annealed by the special technology preventing oxidation [7]. The x-ray analysis

(c) A. B. Beznosov, E. L. Fertman, V. V. Eremenko, P. P. Pal-Val, V. P. Popov, and N. N. Chebotayev, 2001 
have shown that the samples represented homogeneous single-phase solid solutions. The metallographic data point that the alloys represent single phase solid solutions with polyhedral grains of $40-130 \mu$ size. The samples represent rods of $3 \times 3 \times 15 \mathrm{~mm}$ size.

The temperature dependences of elastic modules were measured in the temperature range $5-300 \mathrm{~K}$ by the ultrasonic technique [8] for longitudinal and torsion standing waves at the frequencies $89 \mathrm{kHz}<\mathrm{v}<93 \mathrm{kHz}$ and $54 \mathrm{kHz}<\mathrm{v}<56 \mathrm{kHz}$, respectively. The ultrasonic measurements were accompanied with the measurements of the magnetization in the fields of about 4 Oe by the induction method. The thermal expansion measurements were carried out for the $\mathrm{Gd}_{92.5} \mathrm{Y}_{7.5}$ alloy and pure Gd at temperatures $300-77 \mathrm{~K}$ in zero magnetic field as well as in the transversal external magnetic fields up to $4 \mathrm{kOe}$.

\section{Theoretical aspect}

Spontaneous magnetostriction is caused by dependence of the interactions between magnetic moments in the system on the interatomic distances. The theory of the magnetic structures in the rareearth metals [9-11] requires an including to the system energy at least three force parameters, describing the above mentioned interactions: isotropic exchange integral, two-ions exchange-relativistic anisotropy parameter, and the second single ion anisotropy constant.

In the phenomenology approach framework the spontaneous magnetostriction is determined by the minimization of the Gibbs free energy $\Phi$ with an including the magnetoelastic energy $\Phi_{m e}[12,13]$ :

$$
\Phi=\Phi_{0}+\Phi_{m e} .
$$

Here $\Phi_{0}$ is a system energy in the absence of magnetoelastic coupling; $\Phi_{m e}(T)=\sum_{i} \xi_{i} \eta_{i}^{2}(T) P$ is the magnetoelastic contribution in the independent order parameters approximation; $\xi_{i}$ are the magnetostrictive parameters; $\eta_{i}$ are the order parameters, which we consider as the one component, for the simplicity, and $P$ is a pressure.

The volume spontaneous striction is defined as

$$
\Delta V_{m e}=\left(\frac{\partial \Phi_{m e}}{\partial P}\right)_{T}=\sum_{i} \xi_{i} \eta_{i}^{2} .
$$

From the microscopic point of view the spontaneous magnetostriction of the crystal lattice $c$-axis of the alloys can by expressed as [14]

$$
\Delta c \propto \frac{1}{Y} \frac{\partial J_{e r}}{\partial \ln c} M^{2},
$$

where $M$ is the system magnetization; $J_{e r}=J+\beta$ is an exchange-relativistic coupling parameter $(J$ is an exchange parameter and $\beta$ is a magnetic anisotropy parameter), and $Y$ is the Young modulus. Here we have an exchange and a spin-orbit magnetostriction parameters, $\xi_{\mathrm{ex}} \propto\left(M^{2} / Y\right)(\partial J / \partial \ln c)$, and $\xi_{s o} \propto$ $\propto\left(M^{2} / Y\right)(\partial \beta / \partial \ln c)$, respectively.

The mean field theory for the order parameter in the classic limit gives [13]

$$
\eta=\operatorname{coth}\left(3 \eta \frac{T_{C}}{T}\right)-\frac{T}{3 \eta T_{C}},
$$

where $T_{C}$ is the second order phase transition temperature.

The temperature dependence of the relative linear sizes of magnetic solids as a whole may be expressed as a sum

$$
\frac{\Delta L}{L}=D(T)+\Delta(T)
$$

where $D(T)$ is nonmagnetic Debye contribution:

$$
D(T)=A\left(\frac{T}{\Theta}\right)^{4} \int_{0}^{\Theta / T} \frac{x^{3} d x}{\mathrm{e}^{x}-1},
$$

with the $\Theta$ as the Debye temperature and $A$ as a constant, while the $\Delta(T)$ represents contributions of the magnetic ordering:

$$
\Delta(T)=\sum_{i} \xi_{i} \eta_{i}^{2}(T)
$$

\section{Results}

\section{Elastic modules}

The ultrasonic study have revealed in all the alloys $\mathrm{Gd}_{100-x} \mathrm{Y}_{x}(x=5.5,7.5,10.2,12.0)$ three temperature regions of anomalies, that is in contrast with pure Gd, which has anomalies only at Curie temperature $T_{C}$ and spin-reorientation temperature $T_{s}$. The alloys demonstrate anomalies at Curie temperature region, at $T_{h} \approx 248 \mathrm{~K}$, and in an interval near $100 \mathrm{~K}$ as it can be seen at the Fig. 1, 


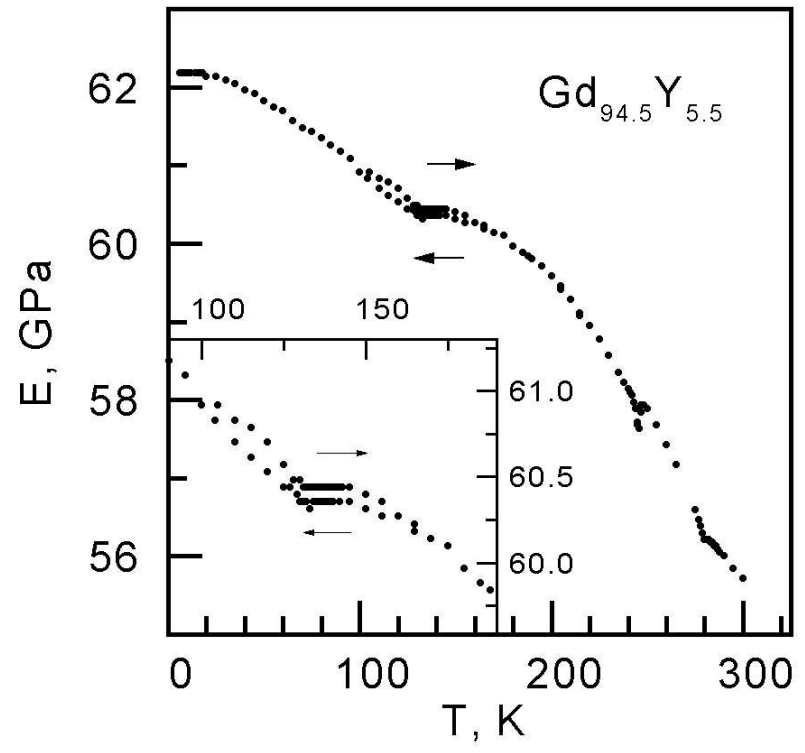

Fig. 1. The Young modulus temperature dependence of the polycrystalline alloy $\mathrm{Gd}_{94.5} \mathrm{Y}_{5.5}$; the arrows show the temperature variation direction. The inset shows the hysteresis at the $\left(T_{s 1}, T_{s 2}\right)$ region.

which represents the Young modulus temperature behavior of the $\mathrm{Gd}_{94.5} \mathrm{Y}_{5.5}$ alloy.

The anomalies at $T_{C}$ temperature are quite ordinary and look like one in pure Gd indicating a typical second order magnetic phase transition.

The anomalies which are located slightly below $T_{C}$ at $T_{h} \approx 248 \mathrm{~K}$ for all the studied alloys are rather unusual. The elastic modules vs temperature curves of all the studied $\operatorname{Gd}_{100-x} \mathrm{Y}_{x}(x \neq 0)$ alloys have rather sharp pits in the narrow temperature range of about $1.5 \mathrm{~K}$ width below $T_{h}$ and have clear first order character (Fig. 1).

The low temperature anomalies represent a plateau of about $10 \mathrm{~K}$ size disposed at the temperatures $146-83 \mathrm{~K}$ for $x=5.5-12$, respectively. It is remarkable, that only the position of the center $\left(T_{s 1}+T_{s 1}\right) / 2$ of the plateau is sensitive to the alloy composition, whereas the size $T_{s 2}-T_{s 1}$ is nearly constant. It means, that the temperatures $T_{s 2}$ and $T_{s 1}$, which we consider as the second order phase transitions points [4], as well as the corresponding order parameters, are coupled. The anomalies have a certain similarity with that in pure $\mathrm{Gd}$ in the region of spin-reorientation transition $T_{s}=226 \mathrm{~K}$, but unlike of pure $\mathrm{Gd}$ the anomalies at $\left(T_{s 2}, T_{s 1}\right)$ region have well defined temperature hysteresis. It should be noted, that there is a temperature hysteresis of the elastic modulus also below the $T_{h}$ point, but of opposite circulation direction of the hysteresis loop [15].

\section{Atomic volume}

The spontaneous magnetostriction, being a volume phenomenon, causes a change of the atomic

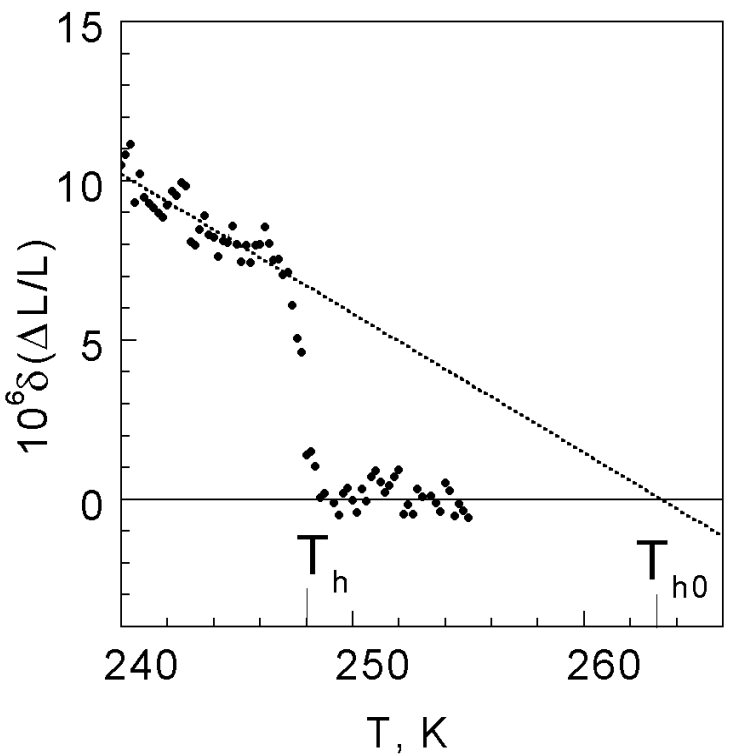

Fig. 2. The «step» $\delta[\Delta L(T) / L]$ of the thermal linear expansion of polycrystalline alloy $\mathrm{Gd}_{92.5} \mathrm{Y}_{7.5}$ in the region of $T_{h}=148 \mathrm{~K}$ obtained by the subtracting of the «background» $T$-dependence from the initial $\Delta L(T) / L$ curve right hand of the $T_{h}$. The $T_{h 0}$ point corresponds to the zero value of the order parameter $\eta_{h}$.

volume $v_{a}$, which is a fundamental parameter of the system. For a polycrystalline sample the linear magnetostriction $\Delta L(T) / L=1 / 3 \Delta v_{a}(T) / v_{a}$ is a convenient value for the experimental determination. The thermal expansion $L(T)$ study has shown, that the temperature behavior of this value in the alloys has complex character, which differs essentially from that of pure $\mathrm{Gd}$, except region in the $T_{C}$ vicinity. There was no lambda-anomaly of the thermal expansion coefficient $\alpha(T)$ at $T<T_{C}$, as it was for pure Gd at spin-reorientation temperature $T_{S}=$ $=226 \mathrm{~K}$. But there was found a step $\delta[\Delta L(T) / T]$ of the $\left[L(T)-L_{0}(T)\right] / L$ function of about $1.5 \mathrm{~K}$ width at $T_{h}=248 \mathrm{~K}$ (Fig. 2), so that the linear thermal expansion coefficient $\alpha(T)$ has nearly symmetrical peak at $248 \mathrm{~K}$. Here $L_{0}(T)=a+b T+c T^{2}$ is a «background» $T$-dependence, determined right hand of the $T_{h} ; a, b$, and $c$ are the fitting constants.

This result confirms the first order nature of the phase transition at $T_{h}$ temperature. The linear extrapolation (dashed line at the Fig. 2) to the zero level of $\delta[\Delta L(T) / T]$ at $T_{h 0}$ defines the zero level of an order parameter of the supposed «initial» second order phase transition, which gives rise to the really observed transition at the $T_{h}$.

Subtracting of the «background» $T$-dependence from the initial $\Delta L(T) / L$ curve right hand of the $T_{s 2}$ gave the negative contribution to the spontaneous magnetostriction caused by the second order phase transitions at $T_{s 2}$ and $T_{s 1}$ (Fig. 3). 


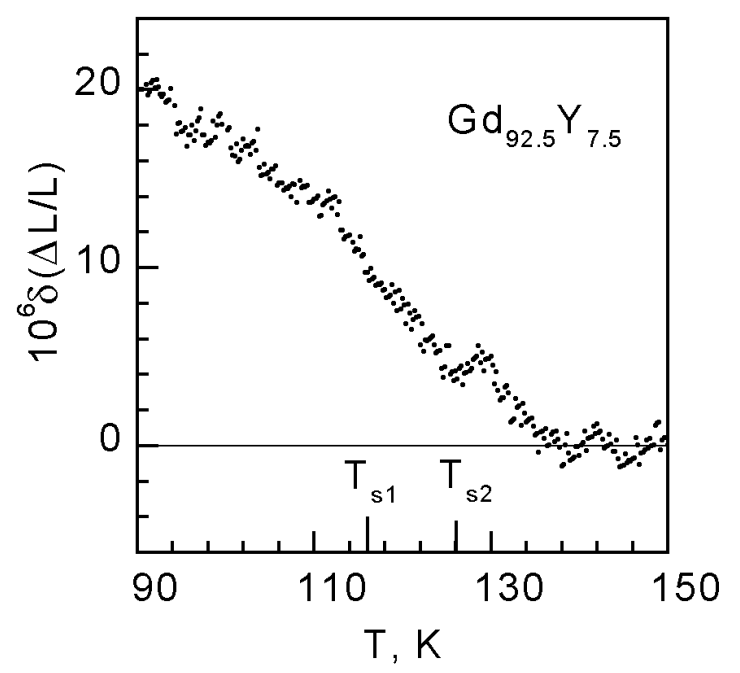

Fig. 3. The phase transitions contribution $\delta[\Delta L(T) / L]$ to the thermal linear expansion of the polycrystalline alloy $\mathrm{Gd}_{92.5} \mathrm{Y}_{7.5}$ in the region of $T_{s 1}, T_{s 2}$ obtained by the subtracting of the «background» $T$-dependence from the initial $\Delta L(T) / L$ curve right hand of the $T_{s 2}$.

The magnetic fields up to $0.26 \mathrm{kOe}$ do not affect sufficiently the $L(T)$ step parameters (neither position, nor height). The field $4 \mathrm{kOe}$ completely suppresses the phase transition at $T_{h}$ (so that $L(T)$ dependence exhibits no peculiarity at all), and turns to a first-order one at $T_{s 3} \approx 0.5\left(T_{s 1}+T_{s 2}\right)$ the two transitions at $T_{s 1}$ and $T_{s 2}$.

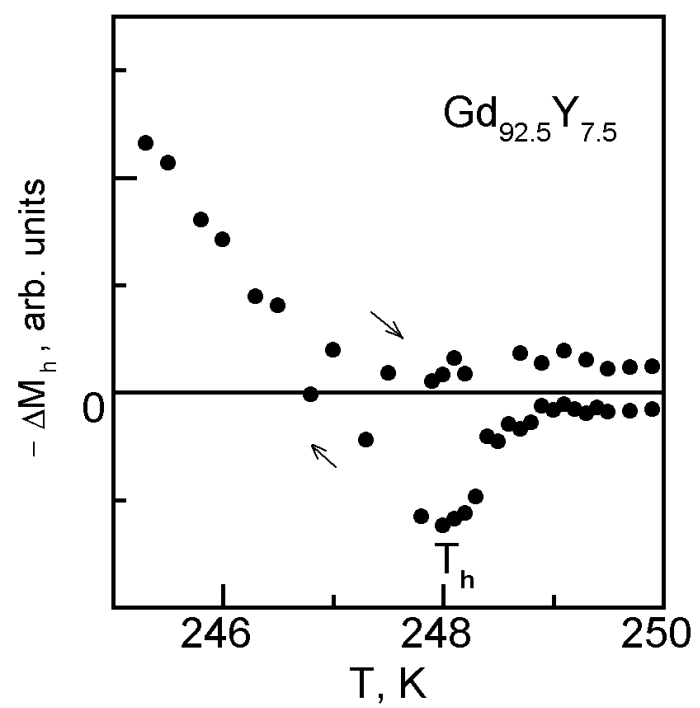

Fig. 4. The negative addition $-\Delta M_{n}$ (for the helical strusture) to the magnetization of $\mathrm{Gd}_{92.5} \mathrm{Y}_{7.5}$ alloy below the $T_{h}$ in arbitrary units; the arrows show the temperature variation direction, and the bar indicates the phase transition point.

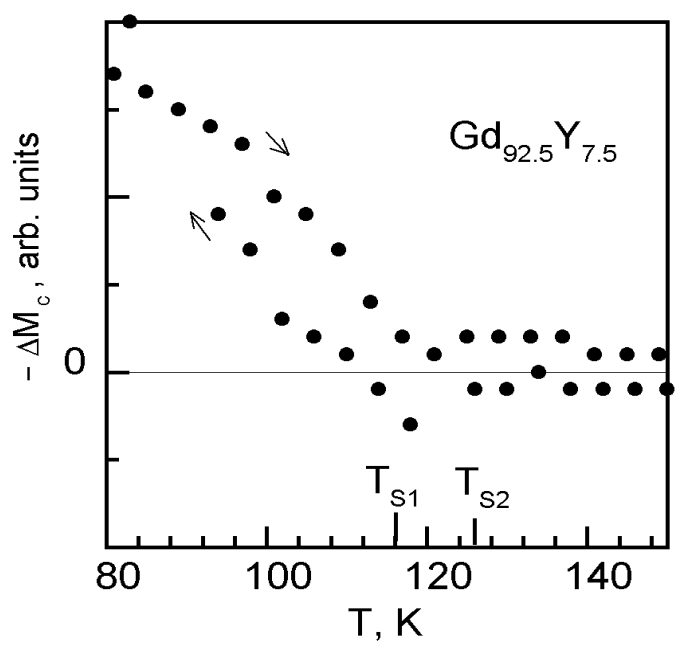

Fig. 5. The negative addition $-\Delta M_{c}$ (for the «conted» structure) to the magnetization of $\operatorname{Gd}_{92.5} \mathrm{Y}_{7.5}$ alloy below the $T_{s 2}$ in arbitrary units; the arrows show the temperature variation direction, and the bars indicate the phase transitions points.

The field effects confirm a strong coupling between the magnetic system and the crystal lattice of the alloys in the studied temperature range.

\section{Spontaneous magnetization}

The spontaneous magnetization $M_{s}$ is one of the components of many components order parameter in the complex magnetic structures. The low field magnetization $M(H)$ can reflect qualitatively the temperature behavior of $M_{s}$. The $M(T ; H)$ measurements have shown the negative contribution to the alloys magnetization left hand of the $T_{h}$ (Fig. 4) as well as below the $T_{s 1}$ point (Fig. 5). Both the $M(T)$ dependences of the studied alloys exhibit temperature hysteresis in the both phase transition regions.

The temperature behavior of the magnetization, as well as that of the above mentioned spontaneous magnetostriction additions, reflects by a certain way the behavior of the order parameters of magnetic phases in the alloys.

\section{Anisotropy constants}

To elucidate the character of the magnetic phases in the alloys an analysis of the temperature behavior of the anisotropy constants $K_{1}, K_{2}$, and $K_{3}$ was performed. The energy of the magnetocrystalline anisotropy was taken in the form 
$E_{a}=K_{1}(T) \sin ^{2}(\theta)+K_{2}(T) \sin ^{4}(\theta)+K_{3}(T) \sin ^{6}(\theta)$,

where the temperature dependence of the $K_{1}, K_{2}$, and $K_{3}$ had the following look [16]:

$$
\begin{gathered}
K_{1}(T)=a \sigma^{2}(T)+b \sigma^{3}(T)+c \sigma^{10}(T)+d \sigma^{21}(T), \\
K_{2}(T)=e \sigma^{10}(T)-f \sigma^{21}(T), \\
K_{3}=0.77 f \sigma^{21}(T),
\end{gathered}
$$

with the relative magnetization $\sigma(T)$ calculated in the molecular field approximation:

$$
\sigma(T)=B_{j}\left[\frac{3 j}{j+1} \frac{\sigma(T) T_{C}+(1-x) h}{T}\right] .
$$

Here $B_{j}$ is the Brillouin function $(j=7 / 2) ; a, b, c$, $d, e, f$ and $h$ are the fitting parameters; $h(1-x)$ is the «short-range order field»; $x$ is the yttrium concentration. The fitting parameters were calculated using the experimental data of the paper [17].

The obtained temperature dependences of the anisotropy constants in the whole temperature range has shown, that the spin reorientation phase transition in the alloys is impossible, at least for the $x \geq 10$ : the $K_{1}$ constant become negative above the Curie temperature (Fig. 6), and the necessary conditions for a mentioned transition are not satisfying for $T_{s 1}<T<T_{C}$. Thus the most probable character of magnetic structure in the range $T_{s 1}<T<T_{h}$ is a sort of the ferromagnetic helix, formation of which is determined jointly by the exchange and anisotropy forces $[9,10]$, in a presence of the random forces caused by the randomly distributed $\mathrm{Y}$ ions in the crystal lattice [3].

\section{Discussion}

\section{The origins of the spontaneous magnetostriction}

The studied magnetic phase transitions in the $\mathrm{Gd}-\mathrm{Y}$ alloys point on a complex character of their electronic structure. The spontaneous magnetostriction in the alloys is forming jointly by the exchange and relativistic forces, and has evidently more items than it is in the simple expression (1) as one can assume regarding the results of the papers $[9,10]$. The strong spin-lattice coupling is the most probable reason for the observed first-order type of the phase transition at $T_{h}$, in contrast with the possible second order transition at $T_{h 0}$. It was shown in the papers $[18,19]$, that the orbital and spin moments of the $5 d$ electrons of $\mathrm{Gd}$ in the metal are strongly correlated, so any change in the local $4 f$ spin structure affected via $4 f-5 d$ exchange and $5 d$ spin-orbit interaction the corresponding change in the ions system. Lack of the local intraatomic exchange spin polarization of the $3 d$ electrons of $\mathrm{Y}$, coupled by the cohesion forces via conduction electrons of $s$ and $d$ types with Gd, leads to the local stresses near the $\mathrm{Y}$ lattice sites. Such random forces affect substantially the magnetic structure of the alloys, destroying the periodic formations in some cases. Hence we can assume, following to the paper [3], that the ferromagnetic cone structure with the random projection of the local magnetic moments in the basal plane is realized for $T_{h}<T<T_{C}$ in the $\mathrm{Gd}-\mathrm{Y}$ alloys, and that such random forces cause a deforming of the helix structure in the region $T_{s 1}<T<T_{s 2}$.

Thus the local stresses caused by the spontaneous magnetostriction near the nonmagnetic $\mathrm{Y}$ ions can substantially affect the character of the phase transition in the $\mathrm{Gd}-\mathrm{Y}$ alloys. We assume, that there is the following sequence of magnetic phase transitions in the studied system: paramagnetic state $\left(T_{C}\right.$, II order $)-$ ferromagnetic cone $(\mathrm{fc})-\left(T_{h}\right.$, I order $)$ - ferromagnetic helix $(\mathrm{fh})-\left(T_{s 2}\right.$, II order transition) - deforming ferromagnetic helix (dh) $-\left(T_{s 1}\right.$, II order transition $)-$ canted ferromagnetic (cf) structure. The last two temperature points correspond to the pair phase transition.

\section{Phase transitions and magnetostriction}

The magnetostriction parameters for all the four magnetic phases ( $\mathrm{fc}, \mathrm{fh}, \mathrm{dh}$, and $\mathrm{cf}$ ) were obtained using the relations (2)-(5) and the experimental $L(T)$ dependences. In all the cases a new order

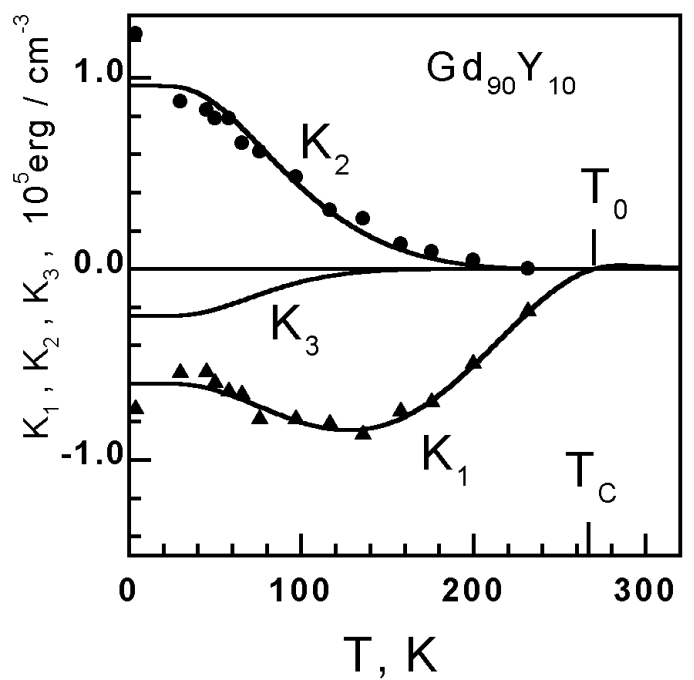

Fig. 6. The anisotropy constants $K_{1}, K_{2}$ and $K_{3}$ of the $\mathrm{Gd}_{90} \mathrm{Y}_{10}$ alloy. 
parameter in the form (2) was determined by the corresponding transition temperature, and the $\xi_{i}$ values were obtained by fitting the dependence: $\delta_{i}[\Delta L(T) / L]=\xi_{i} \eta_{i}^{2}: \xi_{c f}=2.2 \cdot 10^{-5}, \quad \xi_{d h}=$ $=-5.3 \cdot 10^{-5}, \xi_{f h}=7 \cdot 1 \cdot 10^{-5}$ and $\xi_{f c}=1 \cdot 4 \cdot 10^{-3}$. It is easily seen, that the obtained $\xi_{c f}, \xi_{d h}$, and $\xi_{f h}$ values are large enough, comparatively with $\xi_{f c}$, to be valid to affect the more fine magnetic structure than that in the high temperature range $T_{h}<T<T_{C}$. Notice that the exchange-striction model was the first one has been introduced to explain the first order type of the disorder-order magnetic phase transition [20].

\section{Conclusion}

Magnetic phase transitions below the $T_{C}$ are considerably determined by the inner stresses of the crystal lattice caused by the spontaneous magnetostriction effects of the exchange-relativistic nature. Herewith the local random stresses in the vicinity of the $\mathrm{Y}$ ions play an important role assisting in a breakdown of the long-range magnetic order.

The character of the temperature hysteresis of the elastic modules (the clockwise hysteresis loop below the $T_{h}$ and the counterclockwise below the $T_{s 2}$ ) [15] corresponds to the different signs of the spontaneous magnetostriction parameters for the transition between the ferromagnetic cone and the ferromagnetic helix $\left(\xi_{f h}=7.1 \cdot 10^{-5}\right)$, and for the transition region between the ferromagnetic helix and the canted ferromagnetic structure (the sum of $\xi_{d h}=-5.3 \cdot 10^{-5}$ and $\xi_{c f}=2.2 \cdot 10^{-5}$ ).

A. B. B. thanks NATO ASI «Modern Trends in Magnetostriction Study and Application» for the financial Support.
1. H. R. Child and J. W. Cable, J. Appl. Phys. 40, 1003 (1969).

2. S. Bates, S. B. Palmer, J. B. Sousa, G. J. McIntyre, D. Fort, S. Legvold, B. J. Beaudry, and W. C. Koehler, Phys. Rev. Let. 55, 2968 (1985).

3. R. J. Melville, S. B. Palmer, S. Bates, and G. J. McIntyre, J. Magn. Magn. Mater. 116, 267 (1992).

4. A. B. Beznosov, P. P. Pal-Val, and E. L. Fertman, in: Advances in Cryogenic Engineering, 43, Pt. B, Plenum Press, New York (1998), p. 1557.

5. A. B. Beznosov, E. L. Fertman, and P. P. Pal-Val, $J$. Magn. Magn. Mater. 192, 111 (1999).

6. T. Ito, S. Legvold, and B. J. Beaudry, Phys. Rev. B23, 3409 (1981).

7. A. B. Beznosov, V. P. Gnezdilov, V. B. Yeroshenko, N. M. Zvyagina, and G. S. Nikol'skii, Opt. Spectrosc. 42, 1002 (1977).

8. P. P. Pal-Val and H.-J. Kaufmann, Fiz. Nizk. Temp. 2, 325 (1983) [Sov. J. Low Temp. Phys. 9,163 (1983)].

9. T. A. Kaplan, Phys. Rev. 124, 329 (1961).

10. B. R. Cooper, R. J. Elliott, S. J. Nettel, and H. Suhl, Phys. Rev. 127, 57 (1962).

11. S. V. Tyablikov, Metody Kvantovoy Teorii Magnetisma, Nauka, Moscow (1975).

12. K. P. Belov, Magnitnyye Prevrashcheniya, GIFML, Moscow (1959).

13. S. V. Vonsovsky, Magnetism, Nauka, Moscow (1971).

14. A. B. Beznosov and G. S. Nikol'skii, Fiz. Nizk. Temp. 2, 1336 (1976) [Sov. J. Low Temp. Phys. 2, 652 (1976)].

15. V. V Eremenko, A. B. Beznosov, E. L. Fertman, P. P. Pal-Val, and V. P. Popov, in: Advances in Cryogenic Engineering 46, Parts A\&B, Plenum Press, New York (2000), p. 413.

16. M. S. S. Brooks and D. A. Goodings, J. Phys. C Ser. 2, 1, 1279 (1968).

17. K. Tohyama and S. Chikazumi, J. Phys. Soc. Jpn. 35, 47 (1973).

18. A. B. Beznosov, V. P. Gnezdilov, and V. V. Eremenko, JETP Lett. 38, 486 (1983); Fiz. Nizk. Temp. 10, 954 (1984) [Sov. J. Low Temp. Phys. 10, 498 (1984)].

19. A. B. Beznosov, V. V. Eremenko, and V. P. Gnezdilov, J. Magn. Magn. Mater. 43, 243 (1984); ibid. 54-57, 1251 (1986).

20. C. P. Been and D. S. Rodbell, Phys. Rev. 126, 104 (1962). 